\title{
HISTORIA
}

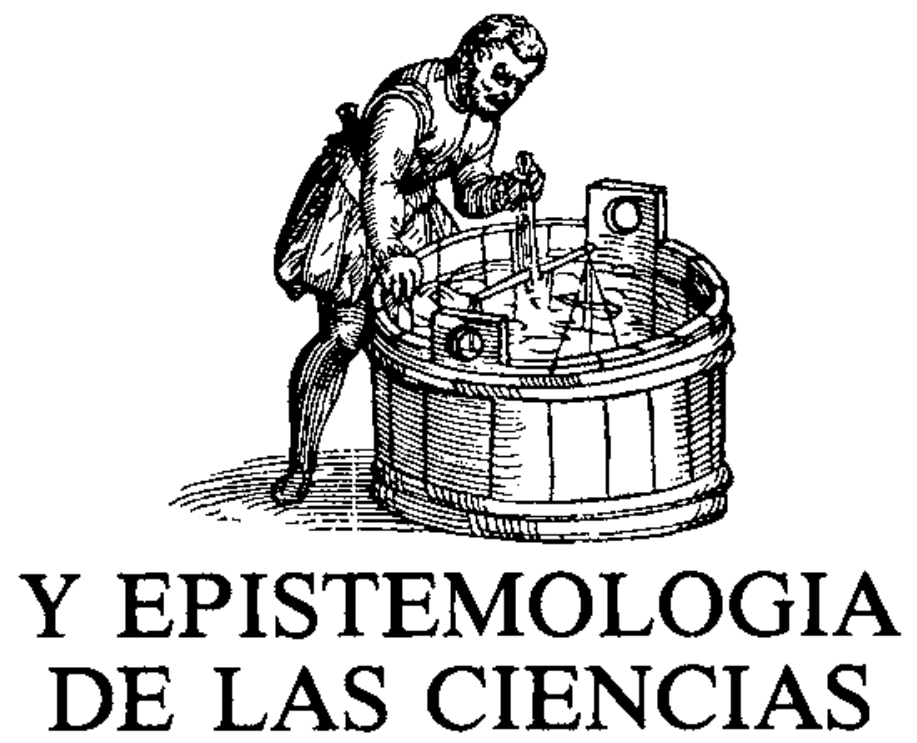

\section{HISTORIA DE LA MATEMÁTICA : INTEGRACIÓN CULTURAL DE LAS MATEMÁTICAS, GÉNESIS DE LOS CONCEPTOS Y ORIENTACIÓN DE SU ENSEÑANZA}

GONZÁLEZ URBANEJA, P.M.

Seminario Permanente de Historia de las Matemátícas. ICE de la Universidad Politécnica de Catalunya. IB «Sant Josep de Calassanç» C/ Sant Quintí, 32-50. 08026 Barcelona.

\section{SUMMARY}

This report seeks to claim a didactic function for the History of Mathematics, justifying the fact that a complete and profound understanding of the fundamental concepts of any science requires the knowledge of its history. The excessive logical-deductive nature of present Mathe.natics has given rise to an excessive dogmatism in its teaching, together with an imposition of exactness and strictness as the fundamental, almost exclusive, values of Mathematics, thus influencing the students' negative response. We believe that these deficiencies can be put back to right by means of the teaching of the History of Mathematics, so as to enrich the teachings culturally. We suggest this subject be integrated inter-disciplinarily into the curriculum, and considered a source of inspiration, permanent self-training, and orientation for all educacional activity. 
"La Ciencia es tanto un hábito de pensamiento como una forma de vida y las Matemáticas son tanto un aspecto de la Cultura como una colección de algoritmos."

Carl B. Boyer (The History of the Calculus and its conceptual development).

\section{INTRODUCCIÓN}

Tanto en la enseñanza media como en la universitaria, he observado en el aula la eficacia pedagógica de las incur* siones en la Historia de las Matemáticas. La capacidad crítica del estudiante no se despierta con la exposición cerrada y acabada de la ciencia estática de los manuales, que, ocultando el sinuoso, zigzagueante y a veces penoso camino de la creación científica, no estimula el desarrollo de valores científicos en el sujeto discente. Las exigencias del curriculo escolar obligaban hasta ahora a cumplit un programa compacto dentro de lo que era el marco cerrado de la asignatura, lo que no propiciaba el contacto con la Historia de la Cienciá perdiendo así la ocasión de dar una visión dinámica de la ciencia, de su evolución y desarrollo, que dé la idea de que es un organismo vivo, en proceso permanente de cambio.

Afortunadamente, las nuevas directrices curriculares van precisamente en sentido contrario, definiendo el currículo como abierto, en el que la asignatura fija, cerrada y obligatoria es sustituida por el crédito flexible, abierto y opcional. Así por ejemplo, en la publicación Disseny Curricular de l'Ensenyament Secundari Obligatori (1989) del Departament d'Ensenyament de la Generalitat de Catalunya, fruto del debate durante cl curso 1987-88, se hace constar:

«II marco curricular para la enseñanza obligatoria establece un diseño que permite unas propuestas curriculares bastante abiertas para permitir adecuaciones diversas y hacer posibles la inclusión de metodologías diferentes.»

Son múltiples las razones para justificar que la Historia de la Ciencia debería influir en la conformación del despliegue curricular en Ios sucesivos niveles de concreción. La Historia de la Ciencia permite advertir qué tipo de problemas dieron lugar a los diversos conceptos, cómo surgieron éstos y cómo fueron evolucionando hasta su estado actual; así se puede dar un significado a la necesaria abstracción de los conceptos científicos. La comprensión completa y profunda de los conceptos fundamentales de una disciplina científica necesita del conocimiento de su historia, ya que ésta pone de manifiesto el proceso dinámico de la actividad científica como desarrollo permanentemente abierto, despertando en el sujeto discente unas actitudes y, sobre todo, unos hábitos metodológigos acordes con el método científico. Además, como escribe Lasa (1984), "la escisión de los saberes, no sólo en dos, sino en mil culturas, hace necesarioel fortalecimicnto de elementos integradores que estimulen la interdisciplinariedad y el reencuentro de los saberes». I.a Historia de Ia Ciencia marca un camino seguro hacia esa reintegración cultural, en particular en la etapa del Bachillerato, que pretende ser integrador, polivalente, flexible y orientador. En la cita- da publicación sobre el diseño curricular se plantea la siguiente pregunta fundamental: "¿De dónde extraer la información para el despliegue curricular de las áreas?" ", es decir: ¿Cuáles deben ser las fuentes del currículo para determinar los contenidos de la enseñanza? Èntre estas fuentes se mencionan la socioantropológica y la epistemológica. Es obvia la incidencia de la Historia de la Ciencia en la obtención de estas fuentes.

\section{LA HISTORIA DE LAS MATEMÁTICAS PARA DESDOGMATIZAR Y ENRIOUECHR CULTURALMENTE LA ENSENANZA DE, LAS MATEMATICAS}

\subsection{El dogmatismo fruto de la tendencia lógico- deductiva.}

El camino de gestación en Matemáticas, particularmente en el último siglo, consiste a menudo en retomar teorías anteriores y refundirlas en un marco nuevo, bajo un enfoque más potente y general que explica mejor (como casos particulares) los resultados ya conocidos y que propicia el descubrimiento de otros nuevos, en la línea de la célebre frase de Alexander Grothendieck, «simplificar generalizando». Tal es el caso, por cjemplo, del proceso de algebrización de la Geometría, tras ci cual las diversas Geometrías Clásicas son meros casos particulares o ejemplos concretos del Álgebra lineal. Como escribe Houzel (1977): «las reelaboracioncs sucesivas que la Matemática hace de las teorías precedentes, atenúan su historia, y aquí hay que buscar una de las principales razones que provocan el que las Matemáticas scan, en un alto grado, negadoras de su propia historia». Cohen (1968) apunta la misma idea basándose en el «carácter acumulativo de la ciencia», que supone que «todo el trabajo útil del pasado esté incorporado a cualquier tratado actual, de modo que se puede prescindir de la obra original». En la introducción de las actas del simpo* sio sobre la Historia de las ciencias y la enseñanza celebrado en Valencia el 18 y 19 de abril de 1980 , Navarro (1980) va más allá al referirse a la actitud de muchos científicos hacia su ciencia, señalando que "situados en la frontera del conocimiento, orgutlosos del carácter innovador de su tarea, muchos sabios ven la reflexión sobre el pasado como una tarea intítil y entorpecedoray.

En décadas pasadas se apoderó de las Matemáticas una irrefrenable tendencia lógico-deductiva. Bourbaki se convirtió en una biblia en la que se encontraba todo cl dogma matemático en forma de axiomas, a partir de los que, mediante un proceso exclusivamente deductivo, independiente de toda experiencia y de toda necesidad, se iban construyendo las estructuras fundamentales de las Matemáticas (estructuras algebraicas, de orden y topológicas) que componían el edificio de toda teoría o rama de la Matemática.

Contra los peligros de este excesivo carácter lógicodeductivo en la Matemática se alzaron algunas voces prestigiosas, preocupadas por desentrañar la esencia de 
esta ciencia y ocupados en mejorar la calidad de la transmisión de su conocimiento. A título de ejemplo mencionemos varias citas de Richard Courant, coautor de la obra Qué es la Matemática, una de las exposiciones más atractivas de las ideas y métodos de la Matemática elemental.

En la introducción de la citada obra Courant y Robbins (1971) manifiestan :

«Parece existir un grave peligro en el excesivo predominio del carácter axiomático deductivo de las Matemáticas (...). Una amenaza seria para la verdadera vida de la Ciencia aparece contenida en la afirmación de que la Matemática no es más que un sistema de conclusiones derivadas de definiciones y postulados que deben ser compatibles, pero que, por lo demás, pueden ser creación de la libre voluntad del matemático. Si esta descripción fuera exacta, las Matemáticas no podrían interesar a ninguna persona inteligente. Sería un juego con definiciones, reglas, y silogismos, sin meta ni motivo alguno (...) Unicamente bajo una disciplina de responsabilidad frente a un todo orgánico, guiada sólo por necesidades intrínsecas, puede la mente libre obtener resultados de valor científico (...) »

También en el prefacio de un texto de análisis matemático redactado en un claro estilo heurístico, Courant y John (1974) insisten nuevamente sobre la peligrosa tendencia que domina la Matemática oficial:

«La Matemática presentada como un sistema de verdades, acabado y ordenado, sin referencia al origen y propósito de sus conceptos y teorías tiene su encanto y satisface una necesidad filosófica. Pero esta actitud introvertida en el campo de la Ciencia no es adecuada para los estudiantes que buscan independencia intelectual más que adoctrinamiento. Menospreciar las aplicaciones y la intuición lleva al aislamiento y a fa atrofiat de la Matemática. Resulta, por consiguiente, extremadamente importante que tanto estudiantes como maestros se resguarden del purismo presumido.»

En el prólogo de la interesante obra de Carl B. Boyer The History of the Calculus and its conceptual developme'nt, Courant es todavía más categórico en torno a la necesidad de conjurar el purismo abstractivo mediante la búsque:da de las ráces históricas de la Matemática (Boyer 1949):

«I os maestros, estudiantes y los amantes del estudio en general, que quieran comprender realmente las fuerzas y las formas de la Ciencia, han de tener alguna comprensión del estado presente del conocimiento como un resultado de la evolución histórica. De hecho, la reacción contra el dogmatismo en la enseñanza científica ha despertado un interés creciente hacia la Historia de la Ciencia. Durante las décadas recientes se han hecho grandes progresos en la investigación de las raíces históricas de la Ciencia en general y de la Matemática en particular.»

Pero no son éstas las únicas voces de alerta. Otros matemáticos interesados por los problemas de la Enseñanza de las Matemáticas lanzaron sus voces de aviso.
Imre Lakatos, en su obra Pruebas y refutaciones. La lógica del descubrimiento matemático, señala con preocupación cómo la metodología euclídea ha desarroliado un estilo necesario de presentación, el estilo deductivista, que comienza enunciando una penosa, artificial y complicada lista de axiomas y definiciones, sin alusión alguna a su origen, a los que siguen teoremas impecablemente demostrados, pero, tan cargados de pesadas condiciones, que parece increíble que alguien los haya podido imaginar. Lakatos (1978) critica con contundencia la situación:

«De acuerdo con el ritual euclídeo, el estudiante se ve obligado a asistir a esta conjura sin hacer preguntas ni sobre el trasfondo ni sobre cómo se realiza el juego de manos (...) Si se pregunta senciliamente cómo es que esas definiciones, esos lemas y el teorema pueden preceder a la prueba, el autor del conjuro to relegará al ostracismo por esta muestra de inmadurez matemática.

"Las Matemáticas se presentan como un conjunto siempre crecjente de verdades eternas e inmutables, en el que no pueden entrar los contraejemplos, las refutaciones o la crítica. El tema de estudio se recubre de un aire autoritario(...) Fl estilo deductivista esconde la lucha y oculta la aventura. Toda la historia se desvanece, las sucesivas formulaciones tentativas del teorema a lo largo del procedimiento probatorio se condenan al olvido mientras que el resultado final se exalta al estado de infabilidad sagrada.»

También Morris Kline, en su popular obra El fracaso de la Matemática moderna, basándose en la evidencia histórica, abunda en incisivos y claros argumentos contra una interpretación puramente deductiva de las Matemáticas elementales. Manifiesta Kline (1978):

«Durante los siglos en los que se edificaron las ramas más importantes de las Matemáticas no había un desarrollo lógico para la mayor parte de ellas. Aparentemente la intuición de los grandes hombres es más poderosa que su lógica (...) Parece claro que primeramente se aceptaron y utilizaron los conceptos que tenían mayor significado intuitivo (...) Los menos intuitivos necesitaron de muchos siglos para su creación o para su aceptación. Además cuando fueron aceptados no fue la lógica lo que indujo a ello a los matemáticos, sino los argumentos por analogía, el significado físico de algunos conceptos y la obtención de resultados científicos correctos.»

Es decir, en general, es la evidencia intuitiva lo que induce a los matemáticos a aceptar los nuevos conceptos. La lógica siempre viene muy por detrás de la invención y suele ser más difícil de alcanzar. Así pues, la Historia de la Matemática sugiere, aunque no lo demuestre, que es más difícil el planteamiento lógico que cl intuitivo. Continúa Kline (1978):

«No se puede dudar de que las dificultades que los grandes matemáticos encontraron son también los obstáculos en los que tropiezan los estudiantes y no puede tener éxito ningún intento de acabar con estas dificultades a base de palabrería lógica.» 
No sólo las dificultades son las mismas sino que los estudiantes deberán superarlas aproximadamente de la misma manera en que fo hicieron los matemáticos a lo largo de la historia, familiarizándose de forma gradual con los nuevos conceptos, trabajando con ellos y apoyándose en toda visión intuitiva que el profesor les vaya dando.

\subsection{Razones históricas para el dogmatismo}

La tendencia a considerar la Matemática como un cuerpo de doctrina cerrado, construido a partir de unas verdades primeras, axiomas o postulados de los que derivan de forma exclusivamente deductiva todo el contenido, ocultando la forma y el camino en que tiene lugar la investigación y el descubrimiento, to es privativa de nuestra epoca, y no se presenta de forma gratuita y arbitraria, sino que es consecuencia de la aparición de problemas de fundamentación y de contradicciones y crisis internas.

La primera manifestacion histórica de estos hechos arranca de la tempestad provocada por el descubrimiento pitagórico de los inconmensurables y de la inquietud que introdujeron en el mutsdo griego las paradojas de Zenón de Elea. $\Lambda$ mbos hechos trajeron consigoel horror al infinito que caracterizó casí toda la producción matemática griega, y que paralizó parcialmente su imaginación creadora, que pasó a segundo plano, a la sombra del supremo rigor lógico impuesto por la escuela platónica, cuyo mayor exponente es la enciclopédica obra de Los Elementos de Fuclides. De este tratado, junto con otros grandes manuales de la Matemática griega, entre ellos Las Cónicas de Apolonio y las obras de Arquírnedes, arranca históricamente el método axiomático-deductivo de exposición que se impondría como modelo. Sólo Pappus, en la pléyade de geómetras griegos, desarrolló una forma de exposición, basada en el método de análisis, que hace patente el camino que sigue la investigación matemática.

EI siglo XVII abre un período que da a luz nuevas ramas de la Matemática (Teoría de Números, el Cálculo de Probabilidades, la Geometría Proyectiva y, sobre todo, la (ieometría Analítica y el Cálculo Infínitesimal), bajo un espíritu innovador, creativo, heurístico y empírico, basado en profundas intuiciones que conducian a la resolución sorprendente y rápida de antiguos y nuevos problemas, aunque en alguno de estos nuevos ámbitos matemáticos, en particular en el Cálculo, la inventiva primaba sobre tos escrúpulos del rigor. La búsqueda de técnicas y métodos heurísticos de rápido descubrimiento cambia el paradigma estilístico de exposición y demostración. Lo que importa ante todo es la consecución de nuevos resultados. Los defectos, ia falta de claridad y las bases inciertas en su fundamentación provocarían críticas (puntillosas como decía Leibniz, pero justificadas), como las vertidas por el Obispo Berkeley, que conducirían a que el Cálculo entrara en crisis. Pero antes de que se creara la adecuada estructura deductiva, los avances a lo largo del siglo XVIII y XIX en Ecuaciones Diferenciales, en Cálculo de Variaciones, en Geometría Diferencial, en Funciones de variable Compleja, etc. fueron gigantescos.
El uso y abuso de la intuición como guía esencial en Ia investigación, produjo a lo largo de los siglos XVII, XVIII y mitad del XIX una relajación en el rigor y una necesidad de esciarecer los fundamentos de las diversas ramas de la Matemática, sobre todo ante la presencia de numerosas paradojas, patologías en la Teoría de Funciones, aparición de las Geometrías no euclídeas, etc. A partir de mediados del siglo pasado, la búsqueda de la consistencia interna de las Matemáticas, en unos nuevos fundamentos más profundos y más seguros, inclinó el péndulo metodológico del lado de la pureza lógica y de la abstracción, imponiéndose el rigor a ultranza como piedra angular de la creación y exposición en la Matemática, de lo que es ejemplar muestra la rigorista sistemática deductiva de Ia moderna síntesis formalista de Bourbaki.

\subsection{Las consecuencias en la enseñanza}

El estilo de Bourbaki que se refleja en gran parte de la literatura matemática, donde se escamotea el proceso histórico de creación, no tarda en imponerse en la enseñanza de la Matemática. Como escriben Malet y Paradís (1984): «De aquella concepción lineal de las Matemáticas surge una enseñanza también lineal. En esencia, se tratará de explicitar, rigurosamente, los axiomas de partida y de demostrar, rigurosamente, los sucesivos resultados que de ellos se derivan. Esto, que es una traición al espíritu real del trabajo matemático, es además una barbaridad pedagógica».

Parece evidente que es preciso conocer la finalidad de ta Matemática para poder comprender sus fundamentos lógicos. Kline (1978) se plantea la siguiente pregunta: «iEs posible entender una teoría sí desde el primer momento se le da la forma definitiva que impone una lógica rigurosa, sin mencionar para nada el camino por el que hallegado a adoptar esta forma? " La contestación es categórica: «No, realmente no es posible entenderla; incluso resulta imposible retenerla si no es de memoria».

La exclusiva interpretación deductiva tiene negativas consecuencias sobre los estudiantes que se sienten engañados al hacerles creer que las Matemáticas han sido creadas por grandes genios que comenzaban con los axiomas y por vía exclusivamente lógica obtenían los teoremas y su demostración impecable. El estudiante, que naturalmente no puede funcionar de esta manera se llega a sentir humillado, acomplejado y desconcertado. Para Kline (1987) ues intelectualmente deshonesto enseñar la interpretación deductiva como si se llegara a los resultados por pura lógica. Esa interpretación destruye la vida y el espíritu de las Matemáticas, no facilita la comprensión y aunque tenga un atractivo estético para $\mathrm{el}$ matemático sirve de anestésico para el estudiante." I a inserción de la Historia de las Matemáticas en la enseñanza de las mismas puede facilitar la comprensión de las dificultades de asimilación. Para Droeven (1980) «el esquema actual de exposición de la enseñanza: axiomasdefiniciones-teoremas-pruebas, sufre de una amnesia histórica, pues al ignorar las génesis históricas de los conceptos matemáticos que involucra, induce a una amnesia conceptual en el alumno, el cual no puede 
reencontrar los obstáculos del conocimiento matemático que han tenido que vencer csos conceptos para presentarse de una manera tan racional y aséptica».

La Matemática, como una de las manifestaciones de la mente humana, refleja la voluntad activa, la razón contemplativa y el deseo de perfección estética; sus elementos básicos son lógica e intuición, deducción y construcción, análisis y sintesis, generalidad y particularidad. La exclusión o infravaloración de algunos de ellos y en particular la imposición del rigor a ultranza como valor fundamental y casi único de la Matemática, ha tenido serias consecuencias en el ámbito escolar. A veces se exige a los estudiantes un rigor que no responde a las necesidades de los problemas que se tratan, de modo que un rigor desorbitado puede hacer que el razonamiento matemático aparente ser un juego absurdo donde se pretende demostrar lo evidente, o peor aún, un complejo entramado, que se plasma en un abstruso lenguaje, don* de se ha difuminado lo que se pretendía aclarar y demostrar. Es curioso que a veces se presenta la peregrina circunstancia (desde el punto de vista del estudiante) de que muchos teoremas son más evidentes que los axiomas y premisas usados para demostrarios. Esto desconcierta al estudiante que puede llegar a pensar que las Matemáticas son un juego de sutilezas inútiles. Tengamos en cuenta en el aula la reflexión de Pascal (1984), en sus Pensamientos: "No intentéis nunca demostrar cosas que son tan evidentes por sí mismas que no existe nada más claro en que podamos apoyarnos para demostrarlas". Así pues, pongamos el rigor en su sitio. El rigor tiene un papel muy importante en las Matemáticas; pero esto sólo afecta al profesional de las Matemáticas. El rigor ha jugado un papel esencial en la fundamentación de la Matemática, pero en este proceso nunca pretendió ser una ayuda básica para la enseñanza. Como indica Kline (1987), «los estudiantes se sienten más atraídos por los frutos que por las raíces de la Matemática», por eso «el rigor puede salvar a las Matemáticas, pero seguramente perderá a los alumnos». El rigor se ha utilizado para dar un aspecto de profundidad a las Matemáticas elementales. Ello ha propiciado un fracaso escolar grave y por tanto un fracaso pedagógico, y no sólo en los niveles elementales y medios, sino también en los universitarios.

Otra de las consecuencias, apuntadas ya en una de las citas de $R$. Courant, es un cierto aislamiento que padece la Matemática en los niveles elementales y secundarios, donde mucha gente no la considera como una disciplina cultural más, sino simplemente como un lenguaje de expresión de las demás ciencias y lo que es más grave como el arma utilizada por el sistema educativo pira filtrar selectivamente al alumnado (esto es patente en las carreras universitarias de tipo técnico), y por ello llega a generalizarse la aversión a esta disciplina, que se contagia de unos a otros. Por todo ello no es extraña la preocupación en cl ámbito docente-matemático por desdogmatizar y enriquecer culturalmente la enseñanza de la Matemática, para reconvertirla en una disciplina cultural en el más amplio sentido de la palabra.

A este fin sirve como instrumento básico la Historia de las Matcmáticas, cs decir, se busca influenciar una metodología de la enseñanza de las Matemáticas mediante
Ia Historia de las Matemáticas y se reclama, por tanto, una función didáctica para ésta.

A pesar de que, como dice Houzel (1977), «está todavía muy extendida la ideología que concibe las ciencias como cuerpos de doctrina universales c intemporales, de modo que por su carácter eterno, las ciencias escaparían a la historia», por fortuna, en la actualidad, muchas personas de formación cientéfica con vocación humanista se interesan por la historia de la disciplina que cultivan, $y$ hace algunos años que ha empezado a abrirse paso una incipiente institucionalización en algunas facultades científicas universitarias españolas de los estudios de Historia de la Ciencia. Como ejemplo significativo citemos el Programa de Master y Doctorado en Historia de las Ciencias organizado por la Universidad Autónoma de Barcelona, así como la introducción de una Historia de la Ciencia y de la Técnica en la Escuela de Ingenieros Industriales de la Universidad Politécnica de Catalunya. Encajaría aquí la opinión que manifiesta Lakatos (1981):

«No podemos tolerar una situación en la que los histo. riadores y fílósofos aprendan matemáticas, ciencia e historia de las exposiciones populares. Ésta es la razón por la que pienso que deberíamos prestar la debida atención al establecimiento de centros de investigación para formar historiadores y filosofos de la ciencia.»

Incluso a nivel de la enseñanza media, parece que la Historia de la Ciencia estará presente, en el currículo secundario, cuando se imponga oficialmente Ia Reforma de las Enseñanzas Medias. No obstante, tal como están las cosas en este momento, la Historia de la Ciencia no está incluida como asignatura específica en el diseño curricular de la enseñanza secundaria postobligatoria, con lo cual si se quisiera introducir debería ser como crédito variable de 30 horas, dependiendo del voluntarismo del profesorado y de las disponibilidades del centro. Como quiera que la administración contempla que el listado de asignaturas específicas pueda ser reajustado, desde algunos ámbitos docentes ya se ha solicitado que la Historia de la Ciencia figure, como asignatura específica, en el currículo de un ampiro sector del alumnado.

\section{LA HISTORIA DE IAS MATEMÁTICAS COMO INSTRUMENTO DIDACTICO, EL METTODO GENETICO}

La forma de utilizar la Historia de las Matemáticas como un instrumento didáctico colaborador puede llevarse a cabo de muy diversas maneras. Se puede, por ejemplo, preceder, mediante una introducción histórica, la exposición de cada tema, situando en la historia los problemas que se van a abordar. Se pueden añadir a los apuntes que se entregan a los alumnos indicaciones, breves resúmenes o notas históricas. Se puede también a lo largo del desarrollo de la clase y en cualquier momento indicar brevemente a qué matemáticos o corriente matemática se debe la introducción de un concepto nuevo, la demostración de un teorema o la resolución de un problema, etc. Asimismo se puede ensayat en aigunos 
temas que se presten a ello, a juicio del profesor, la aplicación del método genético.

El método genético, extraído de la Biología, intenta reconstruir el clima psicológico que envuclve a cada momento creador que haya supuesto un salto cualitativo en la Historia de las Matemáticas. La aplicación de este método en la enseñanza, que ha sido reivindicado por grandes matemáticos y profesores de Matcmáticas, pretende demostrar que, para la perfecta comprensión de un concepto determinado, el alumno ha de repetir a grandes rasgos el proceso histórico que se ha desarrollado hasta la formulación actual del concepto. H. Poincaré (1963), en su obra La ciencia y el mérodo, describe sucintamente la natusaleza del método genético:

«Los zoólogos pretenden que el desarrollo embrionario de un animal resume en un tiempo muy corto toda la historia de sus antepasados desde los tiempos geológicos. Parece que sucede lo mismo en ei desarrollo de los espíritus. Fl cducador debe hacer pasar al niño por donde han pasado sus padres; más rápidamente pero sin saltarse ninguna etapa. De esta manera la historia de la ciencia debe ser nuestra primera guía,»

También Felix Klein, en su interesante texto en dos volúmemes Matemática elemental desde un punto de vista superior, destinado a la formación de los aspirantes al Magisterio, desarrolla una argumentación genética. En efecto, al final del primer volumen Klein (1927) manifiesta:

«Para precisar bien mi opinión en este punto (el punto de vista pedagógico-matemático que aconseja no dar demasiado pronto al alumno cosas demasiado abstractas y difíciles), he de recordar la ley fundamental biogenética, según la cual el individuo en su desarrollo recorre en rápida sucesión todos los estados del desarrollo de la especis a que pertenece (...) Este principio, creo yo, debiera ser seguido también, al menos en sus líneas generales, en la enseñanza de la Matemática lo mismo que en cualquier otra enseñanza; se debería conducir a la juventud, teniendo en cuenta su natural capacidad y disposición, lentamente hasta llegar a las materias elevadas $y$, finalmente, a las formulaciones abstractas, siguiendo el mismo camino por el que la humanidad ha ascendido desde su estado primitivo a las altas cumbres del conocimiento científico (...) Un inconveniente fundamental para la propagación de tal método de enseñanza, adecuadio al alumno y verdaderamente científico es, seguramente, la falta de conocimientos históricos que se nota con sobrada frecuencia».

Otto Toeplitz (a quien se debe un famoso criterio de convergencia de sucesiones) es otro de los creadores del método genético, aplicándolo en uno de los textos más famosos sobre el Cáiculo Infinitesimal y su historia, ta obra The Calculus, a genetic aproach. En el prefacio de la edición alemana (incorporado a la versión americana), su discípulo $\mathrm{G}$. Köthe describe la raturaleza del método genético (T'oeplitz, 1963):

«Ei método genético es la gúa más segura para este ascenso suave (en el estudio del Cálculo), que de otra manera no es fácil de encontrar. Seguid cl curso genético que es el camino que ha seguido el hombre en su comprensión de las Matemáticas, y veréis que la humatnidad ha ido ascendiendo gradualmente desde lo más simple a lo más complejo. Importantes desarrollos ocasionales pueden ser tomados generalmente como indicatdores de progresos metódicos precedentes. Los métodos didácticos pueden beneficiarse enormemente del estudio de la historia.»

En su argumentación contra la interpretación decuctiva Kline se apoya en la evidencia histórica y se adhiere incondicionalmente al método genético. He aquí sus palabras (Kline 1978):

«Cada persona debe pasar aproximadamente por las mismas experiencias por las que pasaron sus antepasados si quiere alcanzar el nivel de pensamiento que muchas generaciones han alcanzado.»

Naturalmente, esta repetición del proceso histórico no debe entenderse al pie de la letra. La construcción de la Ciencia tiene lugar recorriendo, muchas veces de forma tortuosa, caminos que a veces se desandan, de modo que el curso didáctico del desarrollo de la Ciencia no puede tener carácter lineal. Sin ocultar al alumno la forma paulatina y sinuosa de la creación científica, hay que conducirle por caminos rectos, para no hacerle perder tiempo. La aplicación del método genético consiste en contextualizar históricamente, realizando una reconstrucción de la historia que haga patente el conocimiento de las preguntas y necesidades que motivaron on su momento histórico la introducción de un concepto nucvo, asî como las dificultades intrínsecas inherentes al alumbramiento de algunos conceptos y a Ia resolución de algunos problemas, dificultades, que, como señalaba Kline se manifiestan asimismo de forma rotunda en el aprendizaje de los mismos conceptos y en la resolución de los mismos problemas. A título de ejemplo: si los números negativos no aparecicron hasta el milenio de historia matemática, y si fueron necesarios otros mil años hasta que fueran aceptados por Ios matemáticos, podemos estar seguros de que los estudiantes tendrán dificultades con los números negativos. Veamos también cuán significativos son los dos siguientes ejemplos, uno en el ámbito del Álgebra y otro en el del Cálculo. Echando una ojeada a la Historia del Álgebra podremos comprender fácilmente las dificultades que tienen nuestros alumnos de la última ctapa de lá educación básica y la primera de secundaria con las letras que representan las incógnitas: son las mismas dificultades que han padecido los matemáticos durante más de veinte siglos, en el tránsito đesde el Álgebra Retórica de los griegos de la época clásica al Álgebra Simbólica de Vieta (perfeccionàda en cuanto a la notación por Descartes y Newton), pasando por las etapas intermedias del h́lgebra Sincopita de Diofanto de Alcjandría, por los desarrollos de los árabes y por el famoso Arte de la Cosa de los algebristas renacentistas italianos. Análogamente, la historia nos permite entender las terribles dificultades padecidas por nuestros alumnos en la comprensión del concepto de límite y continuidad. Y en este ejemplo el período histórico de dificultades es todavía superior, abarcando prácticamente desde el nacimiento de la Matemática racional 
hasta el pasado siglo, es decir, desde los intentos de escamotear el proceso infinito en Matemáticas, Ilevado a cabo por los griegos mediante el postulado de Arquímedes y el método de exhaución, hasta la reformulación sobre bases rigurosas del nuevo Análisis, emprendida en ef siglo XIX por Cauchy, Weierstrass, Dedekind y otros matemáticos, pasando como etapas intermedias, por las reflexiones de la Escolástica medieval sobre el infirito y el continuo, que fueron propiciando la eclosión durante el siglo XVII de las técnicas y métodos infinitesimales, que, unificadas y generalizadas por Newton y Leibniz, desembocaron en el descubrimiento del Cálculo Infinitesimal por ambos.

\section{LA HISTORIA DE LAS MATEMÁTICAS COMO FUENTE DE INSPIRACION, DE AU- TOFORMACION PERMANENTE Y DE ORIENTACIÓN DELA ACTIVIDAD DOCENTE}

\begin{abstract}
Vamos a comentar ahora la importancia de la Histor.a de las Matemáticas de cara al propio profesor que imparte la disciplina, intentando ponderar la Historia de las Matemáticas como una de las fuentes ineludibles de inspiración en la orientación de la actividad docente del profesor de Matemáticas.
\end{abstract}

El estudio de la Historia de las Matemáticas puede ser un elemento importante en la autoformación permanente del profesor. La enseñanza no es sólo una vocación o una profesión, puede ser también un arte, y es indudable que el conocimiento de la Historia de las Matemáticas con sus grandezas y miserias, con sus momentos estelares y sus épocas oscuras, influirá decisivamente en el espiritu del profesor y en su actitud hacia la propia Matemática, y, como escriben Malet y otros (1983), «bien sabemos que la actitud del profesor hacia la materia que explica es una de las enseñanzas más importantes que transmitimos al alumno».

Las motivaciones para enseñar Matemáticas y desde luego la manera cómo se enseñan pueden verse muy positivamente infuenciadas por esa nueva actitud que crea el conocimiento de la historia. Hay múltiples razones que justifican esta consideración:

\begin{abstract}
A. La perspectiva histórica permite dar una visión más panorámica de los problemas, calibrando con mayor precisión la importancia de los diversos temas, quedando así mejor articulados dentro del contexto de la asignatura.
\end{abstract}

$B$. La Historia de las Matemáticas favorece la comprensión profunda de los problemas matemáticos, a través del conocimiento del proceso real de creación de los conceptos y teorías matemáticos, del contexto en que aparecen, de las ideas que los propician y de las cuestiones que resuelven, de las reformulaciones que sufren, etc., lo que facilita sin duda la comprensión y consideración de las dificultades de los contenidos impartidos.

C. La visión histórica puede apoyar una propuesta de un aprendizaje activo. Citando a Gil (1980), «al extraer de la historia de la ciencia la problemática que, debidamen- te presentada a los alumnos, les permitiera redescubrir, a través de una activiđad investigadora, los conocimientos que la enseñanza tradicional trasmite ya elaborados", se podría tender a alcanzar uno de los objetivos de la enseñanza de cualquier ciencia, a saber, enseñar, en alguna forma, a elaborar ciencia.

$D$. Algunas ideas o creencias no muy ajustadas a la realidad son fácilmente erradicables apelando a la historia. $\Lambda$ este respecto podemos mencionar las siguientes:

- La creencia en la existencia de una Matemática Moderna, desarrollada en la actualidad, contraponiéndose a unas Matemáticas tradicionales, como consecuencia de una profunda revolución. A título de ejemplo digamos que la noción de conjunto, matriz de la presunta Matemática moderna, ya va por el siglo.

- La Matemática que es la más antigua de las ciencias, se la reduce en muchos ámbitos a un mero lenguaje al servicio de las otras ciencias y técnicas. La razón de este reduccionismo tiene su raíz en la aparición de las ciencias experimentales después del Renacimiento, que trajo consigo la asignación de carácter científico a una disciplina sóto cuando había una relación entre una teoría y unos hechos experimentales. I.a Matemática, que anteriormente era considerada comola única ciencia propiamente dicha y que, bajo el prisma filosófico platónico imperante, se la interpretaba como fundada en la intuición intelectual de las esencias preexistentes, al no experimentar directamente sobre la naturaleza, casi se le retiró el carácter de ciencia y se la empezó a considerar como un lenguaje. El carácter científico de las Matemáticas se le restituye profundizando en su historia, advirtiendo que un material experimental de las Matemáticas to constituyen los saberes y doctrinas anteriores sobre los que se va construyendo el edificio de esta ciencia.

- Otra creencia que la historia subvierte es la que supone que el rigor como valor supremo de la Matemática impone una vía única de razonamiento para llegar a los resultados. Como expresa Lakatos (1981): «La enseñanza de la ciencia y de las matemáticas está desfigurada por la usual presentación autoritaria.» Bajo la aplicación rigurosa de estos modos, las clases se vuelven frías, secas y dogmáticas y son estériles para un porcentaje elevado de alumnos. La historia nos muestra cómo se ha llegado a los mismos resultados matemáticos por caminos muy diferentes y no siempre correctos y cómo nuevos modos de razonar se apoyan sobre otros pasados, que deben ser a su ve $z$ modificados para el tratamiento de nuevos problemas.

$E$. Las Matemáticas tienen una fuerza creativa interna que se manifiesta en el devenir histórico y que, como objetivo formativo esencial, debe contribuir a desarroIlar el espíritu creativo del alumno. La exposición aislada de su contexto de simples resultados estereotipados que hay que memorizar, saturados de definiciones abstractas y de demostraciones impecables de teoremas, atrofian la creatividad del alumno, o por lo menos no la favorecen.

$F$. La Historia de las Matemáticas revela los ingentes esfuerzos desplegados por sucesión de generaciones 
matemáticas en la formación de algún concepto nuevo o en la resolución de algún problema importante, que, a la hora de tratarlo en la clase, el profesor con su arrogancia dogmática y autoritaria puede creer, de espaldas a la historia, que debe ser trivial para el alumno. Ya mencionamos antes a título de ejemplo las dificultades intrínsecas del concepto de límite y continuidad o del manejo de las letras en las ecuaciones, pero los ejemplos son múltiples. El profesor que estć al corriente de la historia, adcmás de aprovechar el legado histórico para enriquecer su actividad docente, manifestará una actitud prudente y encontrará sugerencias que faciliten la introducción, sin grandes traumas, de un concepto nuevo, por difícil que sea, y, lo que es más importante, no le desbordará la impacicncia ante las dudas y dificultades de los alumnos, y por consigutente éstos ganarán, además, en confianza y seguridad en sí mismos.

G. La Matemática recreativa se nutre en gran parte de problemas matemáticos que han tenido cierto interés a lo largo de la Historia de la Matemática. Ésta es por tanto un manantial de problemas curiosos que pueden ser tratados de forma lúdica como actividades al margen de la clase y en el marco de las actividades culturales que se organizan periódicamente en los Centros escolares, ya sea a lo largo del curso o concentradas en una Semana Cultural. A pesar de ia escasa audiencia que suelen concitar las Matemáticas, alentadas con anterioridad mediante una propaganda atractiva y presentadas en forma de Taller de Matemática recreativa (González 1989), estas actividades constituyen Experiencias en el Aula (González 1988) que pueden ser un gran señuelo para los alumnos y hacer una sana competencia a las actividades organizadas por los departamentos de Humanidades e incluso colaborando con ellos de forma interdisciplinar, salvando así una cierta incomunicación que existe entre los Seminarios de Ciencias y los Seminarios de Letras, y que transciende necesariamente a los alumnos de una y otra especialidad. Algunos temas que se prestan a ser desarrollados en estos talleres podrían ser la ingente cantidad de curiosidades numéricas, aspectos históricos, aritméticos y geométricos del Teorema de Pitágoras, el omnipresente número de oro y su relación con la Divina Proporción, el famoso número $\pi$, los tres problemas geométricos clásicos (la cuadratura del círculo, la duplicación del cubo y la trisección del ángulo), las inquietantes paradojas sobre el infinito, aspectos artísticos de la Geometría Proyectiva elemental, etc.

Pero también en el marco de la clase pueden encajar coyunturalmente aspectos de la Matemática recreativa. Para Rodríguez (1980), "las inclusiones de asuntos o tratamientos propios de la Matemática recreativa (con sus resultados chocantes con la intuición ordinaria, el rigor suavizado, y la aparición notable de referencias históricas) en los cursos ordinarios son tónicos excelentes que entendemos que ayudan al alumnado a seguir adelante».

H. La Historia de las Matemáticas pone de manifiesto, por una parte, la vinculación entre la Matcmática, ta Filosofia y las Ciencias Sociales, «sirviendo de puente entre la cultura humanística y la científica» como dice Campedelli (1970); así como la relación entre las Matemáticas y sus aplicaciones externas (las Cicncias en general y las diversas técnicas), por otra; y, desde el punto de vista sociológico, permite conocer las fuerzas sociales y productivas que contribuyeron a su desarrollo. Todos estos elementos favorecen la integración interdisciplinar de la Matemática con las demás ciencias particulares, y, más allá de la Ciencia, la integración de la Matemática en el conjunto armónico de los saberes científicos, literarios y artísticos, que constituyen la Cultura. Como dice C.B. Boyer (1949) en el prefacio:

«La Ciencia es tanto un hábito de pensamiento como una forma de vida y las Matemáticas son tanto un aspecto de Ia Cultura como una colección de algoritmos.»

\section{CONCLUSIONES}

Finalizando, en la Historia de las Matemáticas el profesor encontrará un puente de unión entre las ciencias y las humanidades, un medio de autoformación permanente para la comprensión profunda de las Matemáticas, y un instrumento para desarrollar la capacidad de renovación y adaptación pedagógicas. Frente a una metodología lógico-deductivista que conduce a un tratamiento absolutamente dogmático de las Matemáticas, La Historia de las Matemáticas permite plantear activamente el aprendizaje como un redescubrimiento. Como dice Kline (1978): «se puede comprimir la historia y evitar muchos de los esfuerzos y trampas inútiles, pero no es posible darla de lado». Además, la Historia de las Matemáticas es una fuente inagotable de material didáctico, de ideas y problemas interesantes y también, en un alto grado, de diversión y recreo intelectual, en suma, de enriquecimiento personal, científico y profesional, que el profesor debe aprovechar para motivar su labor de transmisión del conocimiento, desdramatizando el aprendizaje de tas Matemáticas. 


\section{REFERENCIAS BIBLIOGRÁFICAS}

BOYER, C.B., 1949. The History of the Calculus and its conceptual development. (Dover: New York).

CAMPEDELLI, L., 1970. Fantasía y Lógica en la Matemática. (Nueva Col. Labor: Barcelona).

COHEN, I.B., 1968. La imaginación humana y la naturaleza, Fronteras del conocimiento. (Eudeba: Buenos Aires).

COLFANT, R. y ROBBINS, H., 1971. ¿Qué es la Matemática? (Agujlar: Madrid).

COURANT, R. y JOHN, E., 1974. Introducción al cálculo y al análisis matemático. (Limusa: México).

DROEVEN, $\mathrm{F}, 1980$. Propuesta para un aprendizaje no ahistorico de las Matemáticas. Actas del Simposio sobre «La Hístoria de las Ciencias y la Enseñanza». (Valencia, abril 1980).

GENERALITAT DE CATALUNYA. DEPARTAMENT D'ENSENYAMENT, 1989. Disseny curricular. Ensenyament Secundari Obligatori.

GIL, D., 1980. Papel de la historia de la ciencia en un planteamiento activo del aprendizaje. Actas del simposio sobre *La historia de las ciencias y la ensenanza». (Valencia, abril 1980).

GONZÁLĽ́, P.M., 1988. Experiencias en el Aula, Comunidad Escolar, 197, p. 14. MFC.

GONZÁLE7, P.M., 1989. Taller de Matemática recreativa, Cuadernos de Pedagogía, 166, pp. 65-66.

HoUZEL, C., 1977. Ponencia de las jornadas: "Historia y Enseñanza delas Matemáticas", Poitiers, 17-18 Junio 1977.
KLEIN, F., 1927. Matemática elemental desde tu punto de visto superior. Vol. I. (Biblioteca Matemática: Madrid).

KLINE, M., 1978. El fracaso de la Matemática moderna. (Siglo XXI: Madrid).

LAKATOS, I., 1978. Pruebas y refutaciones. La lógica del descubrimiento matemático. (Alianza Universidad 206: Madrid).

LAKATOS, I., 1981. Matemáticas, ciencia y epistemología. (Alianza Universidad 294: Madrid).

I.USA, G., 1984. Seminario Permanente de historia de la Matemática, Butletí de la secció de matemàtiques, 16. (Institut d'Estudis Catalans: Barcelona).

MALE'T, A. et al, 1983. Historia de les matemàtiques: cultura i didàctica, Papers de Batxillerat, 3, pp. 74-77.

MALET,A., y PARADIS, J., 1984. Elsorigensil'ensenyament de l'àlgebra simbólica. (Edicions de la Universitat de Barcelona).

NAVARRO, V., 1980. Actas del Simposio sobre «La I fistoria de las Ciencias y la Enseñanza». (Valencia, abril 1980).

PASCAL, B.,1984. Pensamientos. (Ediciones Orbis de Historia del Pensamiento: Barcelona).

POINCARÉ, H., 1963. Ciencia y método. (Esspasa-Calpe, Col. Austral 409: Madrid).

RODRÍGUEZ, A.L., 1980. La hora de la Matemática Recreativa en el Bachillerato actual, Revista de Bachillerato (Cuaderno monográfico $n^{\text {? }} 5$ sobre Matemáticas), pp. 53-56.

TOEPLITZ, O, 1963. The Calculus, a Genetic Approach, (University of Chicago Press: Chicago). 
